\title{
Analysis Tool for Diffusion Tensor MRI
}

\author{
Pierre Fillard ${ }^{1,3}$ and Guido Gerig ${ }^{1,2}$ \\ 1 Department of Computer Science, \\ 2 Department of Psychiatry, \\ University of North Carolina, Chapel Hill, NC 27599, USA \\ 3 ESCPE Lyon, 69100 Villeurbanne, FRANCE \\ \{fillard,gerig\}@cs.unc.edu \\ Software Download: http://midag.cs.unc.edu ${ }^{\dagger}$
}

\begin{abstract}
Diffusion Tensor Imaging (DTI) is becoming a routine magnetic resonance technique to study white matter properties and alterations of fiber integrity due to pathology. The advanced MRI technique needs postprocessing by adequate image analysis and visualization tools. Whereas such tools have been developed at various research centers to drive methodological and clinical research, they have not become widely available as software freely distributed to the community. We have developed an integrated software package for efficient processing, fiber tracking, and interactive visualization of DTI data. This allows even nonexperts to explore DTI data and to obtain results that so far were exclusive to reseach teams with strong computer science support. This report describes our effort to combine common, well-established processing methods for DTI data, a recently developed powerful fiber tracking method and a modern image analysis and visualization environment into an integrated tool.
\end{abstract}

The tensor field caculation is based on an analytical solution of the Stejskal and Tanner's diffusion equation system ([1]). The current version is designed to use the common directional coding proposed by Basser et al. 2]. The vector field defined by the eigenvectors associated with the largest eigenvalues is assumed to represent a good approximation to represent local white matter fiber orientations. The goal of fiber tracking, often called "tractography", is to find likely paths through the vector field between source and target regions of interest (ROIs). We adapted and extended a previously published method 3. The parameters for judging local continuity include minimal FA value, local curvature (angle difference between consecutive vectors), and local coherence (regularization over local neighborhood). This method provides traces with sub-voxel precision. We apply this method with a backward tracking scheme instead of a

\footnotetext{
$\dagger$ This research is supported by the UNC Neurodevelopmental Disorders Research Center HD 03110, the NIH Conte Center MH064065, the Stanley Medical Research Institute, and the Foundation of Hope (Raleigh, NC). We are greatful to Ch. Davatzikos, D. Xu, D. Shen (all University of Pennsylvania), and S. Mori, Johns Hopkins University, for providing an early version of the fiber-tracking code. We thank James MacFall, Duke University, for providing high-resolution DTI test datasets. Color version of paper is available at: http://www.cs.unc.edu/ ${ }^{\sim}$ gerig.
} 
direct tracking. Paths originating from the full brain (target) are traced back to source regions, and only paths passing through these ROIs are finally kept.

The DTI processing package includes the following features:

- Loading input DTI data (GIPL, ANALYZE or DICOM-META format).

- Extraction of tensors and calculation of ADC and FA values.

- 2-D orthogonal slice visualization of DTI data and of ADC and FA images.

- Loading label image with user-defined ROIs.

- Fiber tracking from target to source volumes.

- 3-D interactive visualization of fiber bundles, FA isosurface, source and target ROIs, and user-selected image channels.

- Storing of fiber bundles as sets of poly-lines (ITK data format for curvilinear structures) or as binary fiber-tract label images.

- Storing of FA, ADC image data and 3-D renderings.

This software has been developed in ITK (NLM sponsored Insight Toolkit 4]), a powerfull $\mathrm{C}++$ library dedicated to medical image processing. Currently, the DTI tool is available for Windows PC (Win2000 and XP), Linux, and UNIX Sun Solaris (download at http://midag.cs.unc).
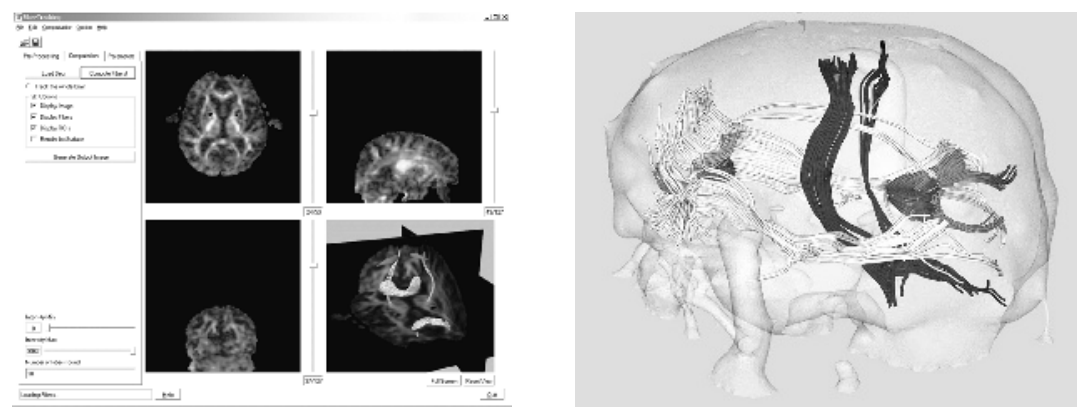

Fig. 1. Left: The DTI processing tool GUI showing 2-D and 3-D visualization options. Right: Result of the reconstruction of 4 major fibers tracts (cortico-spinal tract, traversal tracts through splenium and genu of the corpus callosum, cingulate, longitudinal fasciculi, shown with overlay of the intracranial cavity. DTI are acquired on a GE 1.5T scanner with an EPI sequence and $2 \times 2 \times 2 \mathrm{~mm} 3$ voxel resolution.

\section{References}

1. Westin, C., Maier, S., Mamata, H., Nabavi, A., Jolesz, F., Kikinis, R.: Processing and visulaization for diffusion tensor mri. Medical Image Analysis 6 (2002) 93-108

2. Basser, P., Pierpaoli, C.: Microstructural and physiological features of tissues elucidated by quantitative diffusion tensor MRI. Journal of Magnetic Resonance Imaging (JMRI) 111 (1996) 209-219

3. Xu, D., Mori, S., Solaiyappan, M., van Zijl, P.C.M., Davatzikos, C.: A framework for callosal fiber distribution analysis. NeuroImage 17 (2002) 1131-1143

4. NLM: National Library of Medicine, Insight Toolkit ITK (2002)

http://www.itk.org. 